\title{
Clinical situations requiring radial or brachial access during carotid artery stenting
}

\author{
Damian R. Maciejewski ${ }^{1}$, Łukasz Tekieli ${ }^{1}$, Mariusz Trystuła ${ }^{2}$, Tomasz Tomaszewski ${ }^{3}$, Roman Machnik ${ }^{2}$, \\ Jacek Legutko ${ }^{1}$, Marek Kazibudzki², Robert Musiał ${ }^{4}$, Marcin Misztal ${ }^{2,5}$, Piotr Pieniążek ${ }^{1,2,6}$ \\ ${ }^{1}$ Department of Interventional Cardiology, Institute of Cardiology, Medical College, Jagiellonian University, John Paul II Hospital, Krakow, \\ Poland \\ ${ }^{2}$ Department of Vascular Surgery and Endovascular Interventions, John Paul II Hospital, Krakow, Poland \\ ${ }^{3}$ Department of Neurology and Stroke Unit, John Paul II Hospital, Krakow, Poland \\ ${ }^{4}$ Department of Anesthesiology and Intensive Care, John Paul II Hospital, Krakow, Poland \\ ${ }^{5}$ Department of Coronary Artery Disease and Heart Failure, Institute of Cardiology, Medical College, Jagiellonian University, \\ John Paul II Hospital, Krakow, Poland \\ ${ }^{6}$ Department of Cardiac and Vascular Diseases, Institute of Cardiology, Medical College, Jagiellonian University, John Paul II Hospital, \\ Krakow, Poland
}

Adv Interv Cardiol 2020; 16, 4 (62): 410-417

DOI: https://doi.org/10.5114/aic.2020.101765

\section{A bstract}

Introduction: Radial or brachial access may be preferred in the case of severe peripheral artery disease (PAD) or difficult aortic arch anatomy during carotid artery stenting (CAS).

Aim: To evaluate the clinical conditions indicating potential benefit from non-femoral access as well as feasibility and safety of transradial/transbrachial access (TRA/TBA) as an alternative approach for CAS.

Material and methods: Since 2013, 67 patients (mean age: 70 years old, 44 men, $42 \%$ symptomatic) were selected for CAS with the TRA/TBA approach. The composite endpoint was stroke/death/myocardial infarction within 30 days of the procedure and compared to the propensity score matched transfemoral approach (TFA) group. Clinical (including neurological) examination and Doppler ultrasonography were performed before the procedure, at discharge and at 30 days.

Results: CAS with TRA/TBA was successful in 63/67 patients. Transfemoral access was not feasible due to PAD in 35 (52.2\%) patients, bovine arch in 10 (14.9\%), obesity (BMI > $\left.35 \mathrm{~kg} / \mathrm{m}^{2}\right)$ in $9(13.4 \%)$, severe degenerative disease of the spine in 7 (10.5\%), arch type III in $5(7.5 \%)$ and excessive subclavian stent protrusion in $1(1.5 \%)$ patient. Mean NASCET carotid artery stenosis was reduced from $81 \%$ to $9 \%(p<0.001)$. The composite endpoint occurred in $3(4.8 \%)$ cases and it was not statistically significantly different from the matched TFA group $(6.3 \% ; p=0.697)$. No access site complications requiring surgical intervention or blood transfusion developed.

Conclusions: Transradial and transbrachial CAS may be an effective and safe procedure, and it may constitute a viable alternative to the femoral approach in patients with severe PAD, difficult aortic arch anatomy or obesity.

Key words: peripheral artery disease, carotid artery stenting, radial approach, brachial approach.

Su m m a ry

The femoral artery is the conventional approach for carotid artery stenting (CAS). However, in cases of anatomy variant of the aortic arch and supra-aortic vessels, significant peripheral artery disease or severe obesity, radial or brachial access may be preferred. We found that in these cases transradial and transbrachial CAS may be an effective and safe procedure, and it may constitute a viable alternative to the femoral approach.

\section{Corresponding author:}

Damian R. Maciejewski MD, Department of Interventional Cardiology, Institute of Cardiology, Medical College, Jagiellonian University, John Paul II Hospital, Krakow, Poland, phone: +48 609448 745, e-mail: damianmaciejewski@tlen.pl

Received: 30.04.2020, accepted: 29.06.2020. 


\section{Introduction}

Carotid artery disease is one of the main causes of ischemic stroke and, as a consequence, severe disability in adults [1]. Percutaneous carotid artery stenting (CAS) has become a viable alternative for carotid endarterectomy, particularly in ageing populations with multiple comorbidities and high perioperative risk [2, 3].

The femoral artery is the conventional approach for CAS procedures. However, anatomical variants of the aortic arch and supra-aortic vessels or significant peripheral vascular disease can make catheterization of the common carotid artery difficult or even impossible [4]. In these cases with a challenging aortic arch, the transfemoral approach can lead to prolonged catheter manipulation increasing the perioperative risk of neurological ischemic complications. Furthermore, access site complications as well as contraindications for prolonged bed rest may necessitate an alternative access. Thus, the increasingly used transradial approach for coronary interventions has also been evaluated as an alternative strategy for carotid artery stenting. Carotid artery stenting via the radial/brachial artery is more demanding owing to the need for dedicated systems and techniques. Moreover, operator volume and learning curve are important determinants of technical success and periprocedural outcomes.

\section{Aim}

The aim of the study was to evaluate the clinical conditions indicating potential benefit from non-femoral access as well as feasibility and safety of TRA/TBA as an alternative approach for CAS.

\section{Material and methods}

From June 2013 among 2253 CAS procedures, 67 (3\%) patients were selected to undergo TRA/TBA - CAS. The indications for the non-femoral approach were based on our own experiences and literature review [5-7]. The grade of carotid stenosis and anatomy of the aortic arch were determined by use of preoperative duplex ultrasonography (DUS) and/or computed tomographic angiography (CTA), and verified by intraoperative angiography. Carotid stenting was performed in asymptomatic patients who had carotid artery stenosis $\geq 70 \%$ by arteriography and in symptomatic patients (TIAs or strokes occurring within 6 months prior to CAS) with $\geq 50 \%$ stenosis, according to NASCET (North American Symptomatic Carotid Endarterectomy Trial) criteria [8, 9]. All patients underwent examination by the site neurologist and all provided informed consent before any intervention. This study was approved by the Committee on Research Ethics at our hospital, in compliance with the ethical guidelines of the Declaration of Helsinki. The patients had been treated protractedly with aspirin ( $75 \mathrm{mg} /$ day) and prior to the procedure received a $300 \mathrm{mg}$ loading dose of clopidogrel. After CAS the aspirin was maintained indefinitely and $75 \mathrm{mg} /$ day of clopidogrel was continued for 3 months.

An Allen's test was performed in each patient before the transradial approach. On the procedure day, the right radial or brachial artery was punctured and a $7 \mathrm{Fr}$ Glidesheath Slender (Terumo) was introduced. After the artery cannulation, heparin was given to achieve an activated coagulation time of 250-300 s. Subsequently, Cobra I \& II or Sidewinder diagnostic catheters (Terumo, Cordis) were used and in all cases over a 0.035-inch diagnostic wire a 7 Fr Guider Softip (Boston Scientific) guiding catheter was introduced to the common carotid artery. All CAS procedures were neuroprotected. Direct stenting was performed when possible. Before and after CAS, the degree of stenosis was calculated with quantitative angiography (Figure 1). Technical success was defined as successful revascularization with residual stenosis $\leq 30 \%$ by NASCET criteria. The primary endpoint was the composite of stroke, death and myocardial infarction within 30 days of the procedure. Technical failure was identified when the procedure was abandoned and the cause was noted.

Among 1237 completed TFA CAS we performed propensity score matching with TRA/TBA CAS at a $1: 1$ ratio using the following variables: (i) age, (ii) sex, (iii) clinical presentation, (iv) comorbidities, (v) percentage of carotid artery stenosis, and (vi) contralateral carotid occlusion. Patients after contralateral CAS and re-CAS or procedures using proximal embolic protection devices were excluded from the TFA group.

Clinical (including neurological) examination and DUS were performed in all patients before the procedure, at discharge and at 30 days of follow-up.

\section{Statistical analysis}

Continuous data were presented as means \pm standard deviation; categorical data were given as counts (percentages). Normality of distribution was assessed by the Kolmogorov-Smirnov test. A propensity score matching technique was used to balance available baseline covariates. Fisher' $s$ exact test or $\chi^{2}$ test was used to analyze categorical variables and Student' $s t$-test or Mann-Whitney $U$-test for continuous variables. A $p$-value of $<0.05$ was considered as statistically significant. All analyses were evaluated in Statistica 9.0 (StatSoft Inc) or SPSS 23.0 (SPSS Inc., Chicago, Illinois, USA).

\section{Results}

The mean age of the treated patients (44 men) was 70 years (range: $51-88$ years), and 28 (41.8\%) patients were symptomatic (TIA or stroke occurring within 6 months prior to CAS). Transfemoral access was not feasible due to peripheral artery disease (PAD) in 35 (52.2\%) 

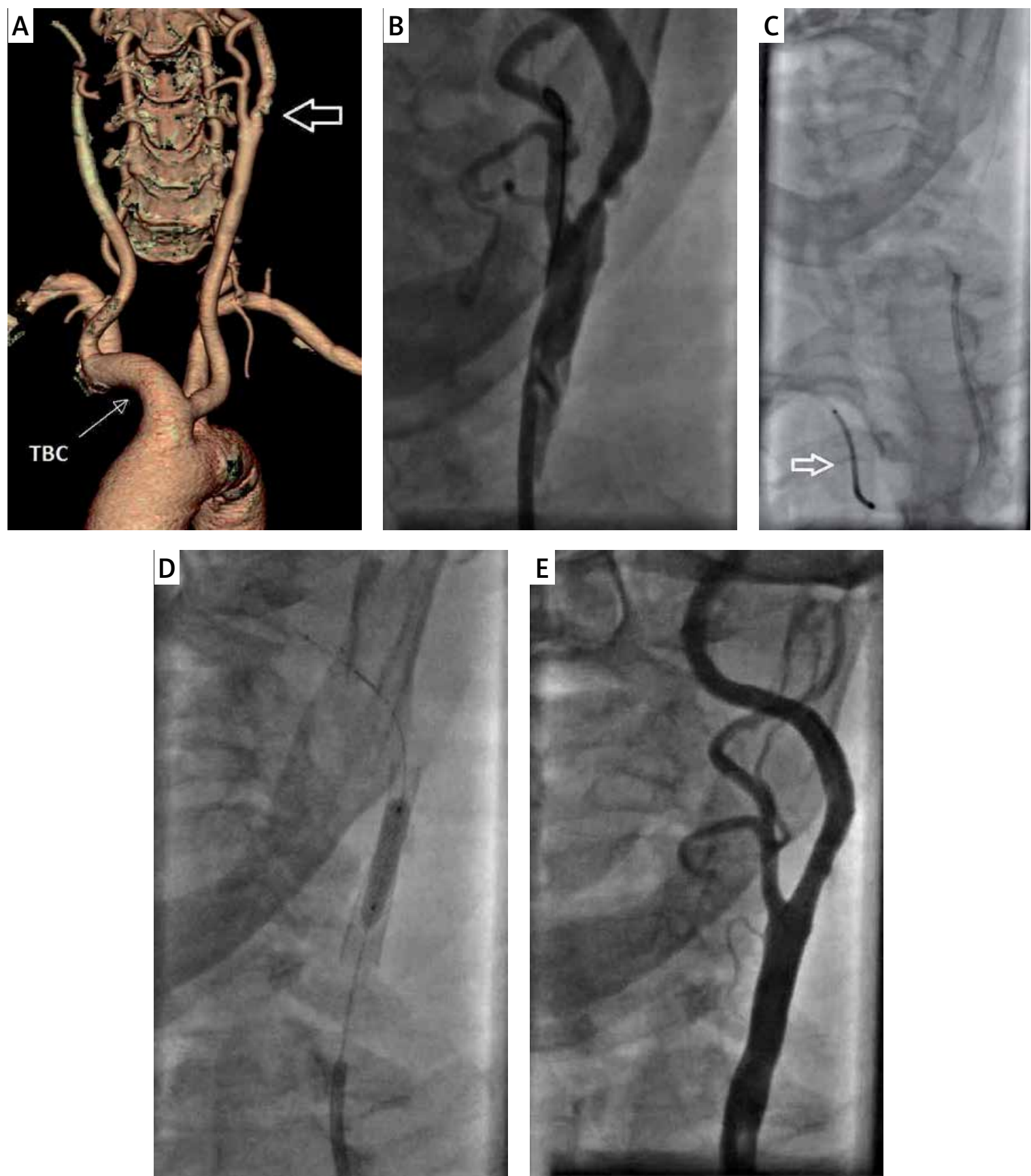

Figure 1. A - Left internal carotid artery stenosis (large arrow). TBC - truncus brachiocephalicus and bovine arch (thin arrow). B - Selective angiography confirming critical stenosis of the left internal carotid artery. C - Self-expanding Carotid Wallstent (Boston Scientific) $7 \mathrm{~mm} \times 30 \mathrm{~mm}$ delivery (white arrow). D - Ultra Soft (Boston Scientific) $5.0 \mathrm{~mm} \times 20 \mathrm{~mm}$ balloon post-dilatation. E - Final angiography of carotid stenting

patients - the most common causes were Leriche syndrome or history of aorto/femoro-femoral bypass implantation. The bovine arch (the left common carotid artery arises from brachiocephalic trunk) was the reason for a radial approach in 10 (14.9\%) cases. Nine (13.4\%) patients were disqualified from TFA CAS due to obesity with a body mass index over 35 and 7 (10.5\%) due to severe degenerative disease of the spine (inability to undergo femoral arterial puncture and/or lie down during/ after procedure). Moreover, type III aortic arch (all three supra-aortic vessels arise before and below the top of the aortic arch) were described in 5 (7.5\%) cases. In 1 (1.5\%) patient, CAS was performed via the radial artery due to excessive protrusion of the stent into the aortic arch after left subclavian artery stenting in the past (Figure 2). Brachial access was used in 3 cases due to an unsuccessful radial artery puncture attempt and in one case of radial artery occlusion. Baseline demographic and clinical 

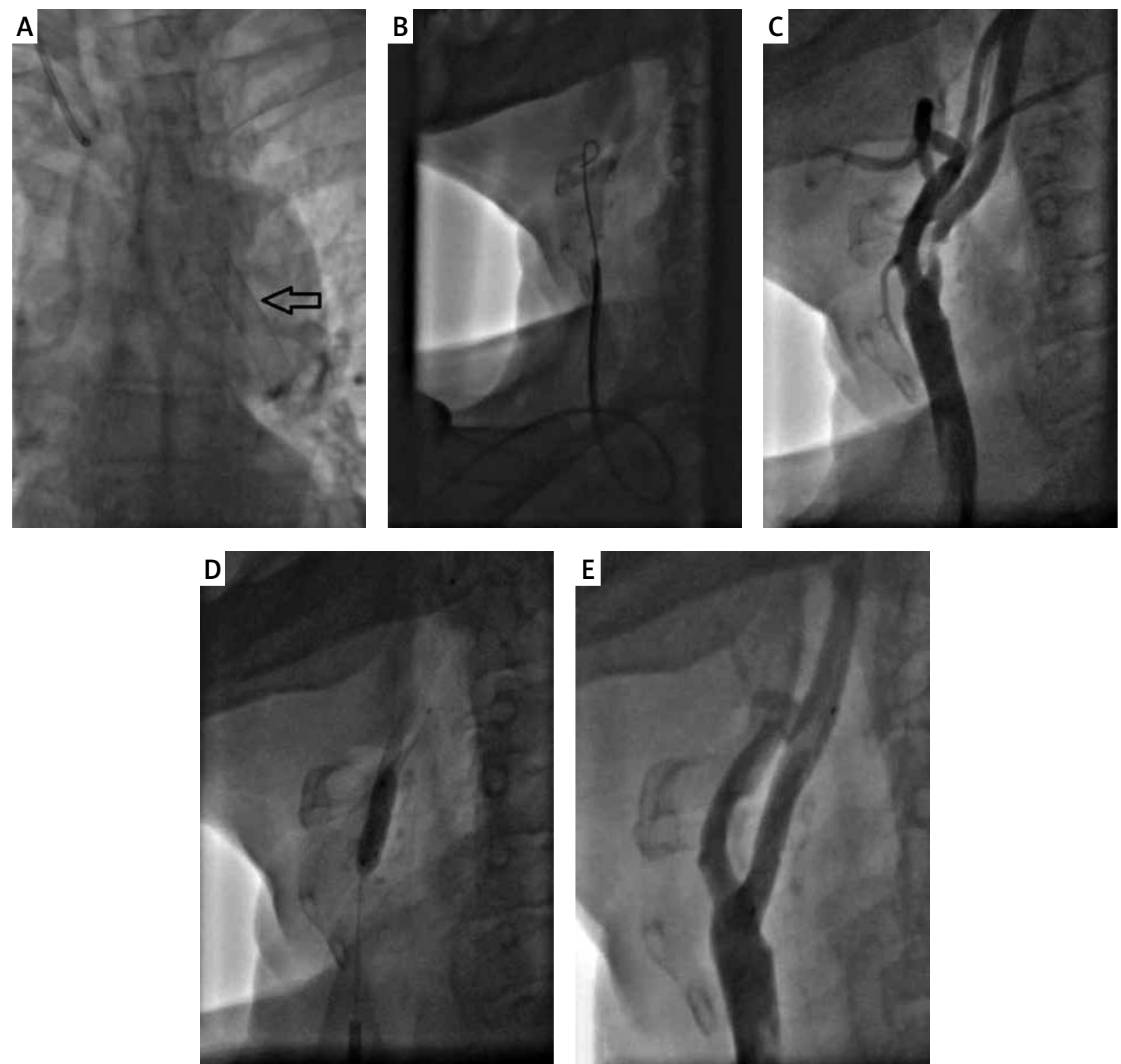

Figure 2. A - Excessive protrusion of subclavian stent into the aortic arch (black arrow). B - Successful cannulation of the right common carotid artery with $7 \mathrm{Fr}$ Guider Softip (Boston Scientific) guiding catheter via the right radial artery. $\mathbf{C}$ - Selective angiography confirming critical stenosis of the right internal carotid artery. D - Self-expanding Precise (Cordis) $8 \mathrm{~mm} \times 30 \mathrm{~mm}$ post-dilatation with $5.0 \mathrm{~mm} \times 20 \mathrm{~mm}$ balloon. E - Final angiography showing optimal effect of RICA-CAS

characteristics, indication for CAS and propensity score matching with the TFA group are listed in Table I.

We were able to successfully complete the CAS procedure using transradial/transbrachial access in 63 of 67 patients (94\% technical success rate). Procedural failure was due to unsuccessful distal embolic protection device delivery, massive carotid artery calcification and subclavian artery tortuosity in 2 cases. In 2 of these 4 patients the procedure was completed through a direct transcervical approach without complications (the puncture sites were closed with sutures). The other 2 patients were qualified for high-risk carotid endarterectomy (both were uneventful). The mean NASCET carotid artery stenosis was reduced from $81 \%$ to $9 \%(p<0.001)$. Direct stent implantation was performed in 30 (47.6\%) cases. Among 63 completed TRA/TBA CAS, technical success was achieved in 61 (97\%) patients. In 2 patients 35-40\% residual stenosis remained without peri- and postprocedural complications. Procedural data are shown in Table II.

The composite endpoint occurred in $3(4.8 \%)$ cases. These patients were disqualified from TFA CAS due to $P A D$, degenerative disease of the spine and severe obesity. One patient died as a result of an intracranial hemorrhage that occurred 2 days after the procedure. Two 
Table I. Patient baseline characteristics and indications for radial/brachial access $(n=67)$. Propensity score matching between TRA/TBA and TFA CAS at $1: 1$ ratio

\begin{tabular}{|c|c|c|c|}
\hline Parameter & \multicolumn{3}{|c|}{ Value } \\
\hline \multicolumn{4}{|l|}{ Demography and comorbidities: } \\
\hline Age [years] & & $70 \pm 8.6$ & \\
\hline Gender, men & & $44(65.7)$ & \\
\hline Stroke/TIA & & $28(41.8)$ & \\
\hline Hypertension & & $66(98.5)$ & \\
\hline Diabetes mellitus & & $21(31.3)$ & \\
\hline Dyslipidaemia & & $66(98.5)$ & \\
\hline Coronary artery disease & & $43(64.2)$ & \\
\hline Previous percutaneous intervention coronary & & $24(35.8)$ & \\
\hline Previous myocardial infarction & & $16(23.9)$ & \\
\hline History of coronary artery bypass grafting & & $9(13.4)$ & \\
\hline \multicolumn{4}{|l|}{ Indication for radial/brachial access: } \\
\hline Severe peripheral artery disease & & $35(52.2)$ & \\
\hline Bovine arch & & $10(14.9)$ & \\
\hline Obesity, BMI > $35 \mathrm{~kg} / \mathrm{m}^{2}$ & & $9(13.4)$ & \\
\hline Severe degenerative disease of the spine & & $7(10.5)$ & \\
\hline Type III aortic arch & & $5(7.5)$ & \\
\hline Excessive protrusion of the subclavian stent into the aortic arch & & $1(1.5)$ & \\
\hline \multicolumn{4}{|l|}{ Propensity score matching } \\
\hline Parameter & TRA/TBA $(n=63)$ & TFA $(n=63)$ & $P$-value \\
\hline Age [years] & $69.8 \pm 8.7$ & $69.2 \pm 8.6$ & 0.687 \\
\hline Gender, men & $41(65.1)$ & $43(68.3)$ & 0.705 \\
\hline Stroke/TIA & $26(41.3)$ & $25(39.7)$ & 0.856 \\
\hline Diabetes mellitus & $19(30.2)$ & $23(36.5)$ & 0.450 \\
\hline Previous percutaneous intervention coronary & $23(36.5)$ & $25(39.7)$ & 0.714 \\
\hline Previous myocardial infarction & $15(23.8)$ & $15(23.8)$ & 1.000 \\
\hline History of coronary artery bypass grafting & $9(14.3)$ & $8(12.7)$ & 0.794 \\
\hline Contralateral carotid occlusion & $11(17.5)$ & $10(15.9)$ & 0.811 \\
\hline Percentage of carotid artery stenosis & $81.5 \pm 8.2$ & $81.7 \pm 8.1$ & 0.744 \\
\hline
\end{tabular}

Values are mean \pm SD or $n(\%)$.

patients sustained a major ischemic stroke during the first postprocedural day. Both subsequently improved substantially over the next few weeks. There was no significant difference in complication rate between bovine arch, type III aortic arch, obesity, degenerative disease of the spine and PAD subgroups.

No radial or brachial access site complications requiring surgical intervention or blood transfusion developed. Although radial artery patency assessment was not per- formed after the procedure, no hand ischemia symptoms occurred. Neither TIA nor myocardial infarction occurred.

In the matched TFA group, the composite endpoints occurred in 4 (6.3\%) cases - 2 major ischemic strokes and 2 TIAs. No statistically significant differences were observed in the overall endpoints (4.8\% vs. 6.3\%; $p=0.697)$, major stroke (3.2\% vs. $3.2 \% ; p=1.000)$, TIA ( $0 \%$ vs. $3.2 \% ; p=0.154)$ or death $(1.6 \%$ vs. $0 \% ; p=0.315)$ between TRA/TBA and the matched TFA groups. 


\section{Discussion}

The transfemoral approach (TFA) is the most common access for carotid angiography and carotid artery stenting as the majority of operators are familiar with this technique, it accepts the use of large diameter introducers (typically $9 \mathrm{~F}$ for a proximal neuroprotection device) and is associated with a high technical success rate $[10,11]$. Despite these advantages, the femoral approach has many limitations including problematic carotid cannulation in patients with a type III aortic arch or with a bovine arch. As reported by Nagarra et al., unfavorable aortic arch anatomy was an independent risk factor of periprocedural complication of CAS [12]. Faggioli et al. suggested that during transfemoral CAS, silent cerebral embolization (assessed with diffusion-weighted $M R$ ) is related not only to the stenting procedure but also to aortic arch catheter maneuvering - 58\% patients had signs of ischemia in both hemispheres [13]. Therefore, the transradial approach may reduce catheter-induced embolization from the aortic arch by the avoidance of catheter manipulation [14].

Obesity, a well-known major public health issue, is an independent risk factor for cardiovascular disease (CVD) and one of the main causes of the increased risk of polyvascular atherosclerosis [15]. Ahmed's meta-analysis reported the prevalence of asymptomatic carotid stenosis $>70 \%$ in patients with lower extremity artery disease (LEAD) at 14\% [16]. Moreover, in Aboyans's study, carotid disease appears to be twice as common among LEAD patients as among coronary artery disease patients [17]. Both obesity and LEAD are associated with more access site and bleeding complications after TFA interventions. Thus, in cases of severe obesity, aortoiliac disease or unfavorable aortic arch anatomy transradial access (TRA) remains a viable alternative. TRA was first introduced by invasive cardiologists and has become the first coronary access line due to the lower vascular/systemic complication rate and number of major bleeding events. In addition, fast mobilization after the TRA procedure results in increased comfort and shortens hospitalization time $[18,19]$. Thus, TRA has also been proposed for the treatment of supra-aortic vessels, including extracranial carotid arteries $[5,20]$. Compared with transradial coronary interventions, CAS via the radial/brachial artery is more challenging due to the need for use of dedicated systems and techniques, and it demands the highest level of experience from operators. Furthermore, the small size of the radial artery may exclude the use of large-diameter devices ( $>$ FF), including proximal embolic protection systems.

Carotid artery stenting by the TRA/TBA may be performed using the contralateral or the ipsilateral radial artery. However, the right TRA/TBA may be the preferred strategy for cannulation of the right common carotid artery (CCA) or the left CCA, especially in the presence of
Table II. Procedural data $(n=67)$

\begin{tabular}{lc} 
Parameter & Value \\
\hline Right carotid artery & $39(58.2 \%)$ \\
\hline Left carotid artery & $28(41.8 \%)$ \\
\hline Contralateral occlusion & $11(16.4 \%)$ \\
\hline Technical success rate by radial/brachial access: & 63 \\
\hline Transradial access & 59 \\
\hline Transbrachial access & 4 \\
\hline Direct stenting & $30(47.6 \%)$ \\
\hline Angiographic stenosis evaluation (\%): & $81.5 \pm 8.2$ \\
\hline Pre-intervention & $9.1 \pm 7.6$ \\
\hline Post-intervention & $33(52.3 \%)$ \\
\hline Stent type: & $18(28.6 \%)$ \\
\hline Carotid Wallstent (Boston Scientific) & $6(9.5 \%)$ \\
\hline Roadsaver (Terumo) & $3(4.8 \%)$ \\
\hline Cristallo Idealle (Medtronic) & $3(4.8 \%)$ \\
\hline Precise (Cordis) & $29(46 \%)$ \\
\hline Mer (Balton) & $19(30.2 \%)$ \\
\hline Neuroprotection type: & $9(14.3 \%)$ \\
\hline Spider FX (Medtronic) & \\
\hline Emborion (Allium Medical) & \\
\hline
\end{tabular}

a bovine arch. The advantage of the right radial/brachial approach is an adequate support (even at acute angles of the common carotid artery origin) and lower radiation exposure. TRA for non-bovine left internal carotid disease is more challenging due to the unfavorable origin angle resulting in insufficient support for the catheter system [21]. Keeping in mind all the above, we concluded that with optimal selection of cases, right radial/brachial artery access CAS may by successful in all cases.

The well-known benefits of radial access CAS are fewer vascular access complications and major bleeding events when compared with the femoral approach, especially in the older population [6]. Despite the fact that TBA has been shown to be associated with relatively high risk of vascular complications [22], in our study no access-site complications requiring surgical intervention or blood transfusion occurred.

Cerebral hyperperfusion syndrome (CHS) after carotid artery revascularization is a rare $(<1 \%)$ but potentially devastating complication of CAS leading to an intracranial hemorrhage $(\mathrm{ICH})$ and death in up to $50 \%$ of cases [23-25]. Preoperative risk factors for development 
of $\mathrm{CHS} / \mathrm{ICH}$ include long standing hypertension, diabetes mellitus, advanced age, recent procedure on contralateral vessel (< 3 months), high degree of stenosis with poor collateral flow, severe contralateral carotid occlusion, recent stroke and/or ischemia or incomplete circle of Willis [26]. In our study, the patient who died as a result of an intracranial hemorrhage had many of the above risk factors - long-standing hypertension with severe obesity $\left(\mathrm{BMI}>35 \mathrm{~kg} / \mathrm{m}^{2}\right)$, diabetes mellitus, recent stroke and $90 \%$ stenosis of the carotid artery with contralateral occlusion. In our case, ICH occurred 2 days after the procedure and it is consistent with the data showing that the average time from the CAS procedure to development of CHS was $12 \mathrm{~h}$ and no cases of CHS were described after 6 days [27]. The current practice is to discharge patients within 24-48 h of CAS; therefore they should be aware of the possibility of developing CHS after returning home.

Khan et al. identified multiple risk factors (clinical, institutional, angiographic and procedural) for stroke, death, and myocardial infarction (MI) within 1 month in patients undergoing carotid artery stent placement [28]. Distal embolization is the major neurological complication occurring after CAS, is not only limited to the procedural time and may occur at any stage of the procedure (including introduction/retrieval of the guiding catheter) $[29,30]$. The use of novel, mesh-covered stents has revealed an important role of exclusion of atherosclerotic plaque from the circulation [31, 32], but their use does not guarantee full anti-embolic protection during CAS. In our group, two major ischemic strokes occurred within a few hours after CAS. Both patients had angiographic and clinical risk factors for peri- and postprocedural stroke (symptomatic status, diabetes mellitus, chronic kidney failure). Despite usage of a closed-cell designed stent (Roadsaver (Terumo) and Carotid Wallstent (Boston Scientific)) it was impossible to avoid neurological embolic complications in those patients.

The percentage of endpoints in TRA/TBA and the matched TFA groups was similar without statistically significant differences. It shows that despite the additional factors disqualifying from the femoral approach, TRA/ TBA may constitute a viable alternative in these high-risk patients.

There are several important limitations of the study. First, this is a nonrandomized, retrospective observational registry involving a small group of selected patients. The second limitation is that microembolization was not assessed with imaging tests, and thus silent cerebral ischemia might have been overlooked.

\section{Conclusions}

Transradial and transbrachial CAS may be an effective and safe procedure, and it may constitute a viable alternative to the femoral approach in patients with severe peripheral artery disease, difficult aortic arch anatomy or obesity.

\section{Conflict of interest}

PP has received travel reimbursements for attending conferences, research grant funding, lecture honoraria, speakers fees from Abbott, Balton, Boston Scientific, Medtronic and Terumo. None of the other authors has competing interests to declare.

\section{References}

1. Barnett HJM, Gunton RW, Eliasziw M, et al. Causes and severity of ischaemic stroke in patients with internal carotid artery stenosis. JAMA 2000; 283: 1429-36.

2. Brott TG, Hobson RW, Howard G, et al. Stenting versus endarterectomy for treatment of carotid-artery stenosis. N Engl J Med 2010; 363: 11-23.

3. Silver FL, Mackey A, Clark WM, et al. Safety of stenting and endarterectomy by symptomatic status in the Carotid Revascularization Endarterectomy Versus Stenting Trial (CREST). Stroke 2011; 42: 675-80.

4. Berko NS, Jain VR, Godelman A, et al. Variants and anomalies of thoracic vasculature on computed tomo-graphic angiography in adults. J Comput Assist Tomogr 2009; 33: 523-8.

5. Etxegoien N, Rhyne D, Kedev S. The transradial approach for carotid artery stenting. Catheter Cardiovasc Interv 2012; 80: 1081-7.

6. Ruzsa Z, Nemes B, Pinter L. A randomized comparison of transradial and transfemoral approach for carotid artery stenting: RADACAR (Radial Access for Carotid Artery Stenting) study. Eurolntervention 2014; 10: 381-91.

7. Maciejewski D, Pieniążek P, Tekieli $Ł$, et al. Transradial approach for carotid artery stenting in a patient with severe peripheral artery disease. Adv Interv Cardiol 2014; 10: 47-9.

8. North American Symptomatic Carotid Endarterectomy Trial Collaborators. Beneficial effect of carotid endarterectomy in symptomatic patients with high-grade carotid stenosis. N Engl J Med 1991; 325: 445-53.

9. Asymptomatic Carotid Atherosclerosis Study Group. Endarterectomy for asymptomatic carotid artery stenosis. JAMA 1995; 273: 1421-8.

10. Pieniazek P, Musialek P, Kablak-Ziembicka A, et al. W. Carotid artery stenting with patient- and lesion-tailored selection of the neuroprotection system and stent type: early and 5-year results from a prospective academic registry of 535 consecutive procedures (TARGET-CAS). J Endovasc Ther 2008; 15: 249-62.

11. Pieniazek P, Tekieli L, Musiałek P, et al. Carotid artery stenting according to the tailored-CAS algorithm is associated with a low complication rate at 30 days: data from the TARGET-CAS study. Kardiol Pol 2012; 70: 378-86.

12. Naggara O, Touze E, Beyssen B, et al. Anatomical and technical factors associated with stroke or death during carotid angioplasty and stenting: results from the Endarterectomy Versus Angioplasty in Patients with Symptomatic Severe Carotid Stenosis (EVA-3S) trial and systematic review. Stroke 2011; 42: 380-8.

13. Faggioli G, Ferri M, Rapezzi C, et al. Atherosclerotic aortic lesions increase the risk of cerebral embolism during carotid stenting in patients with complex aortic arch anatomy. J Vasc Surg 2009; 49: 80-5. 
14. Barbato JE, Dillavou E, Horowitz MB, et al. A randomized trial of carotid artery stenting with and without cerebral protection. J Vasc Surg 2008; 47: 760-5.

15. Barroso TA, Marins LB, Alves R, et al. Association of central obesity with the incidence of cardiovascular diseases and risk factors. Int J Cardiovasc Sci 2017; 30: 416-24.

16. Ahmed B, Al-Khaffaf H. Prevalence of significant asymptomatic carotid artery disease in patients with peripheral vascular disease: a meta-analysis. Eur J Vasc Endovasc Surg 2009; 37: 262-71.

17. Aboyans V. Polyvascular disease: definition, epidemiology, relevance. In: Pan Vascular Medicine. Lanzer P (ed.). Springer, Berlin 2015; 4779-810.

18. Le Corvoisier P, Gellen B, Lesault PF, et al. Ambulatory transradial percutaneous coronaryintervention: a safe, effective, and cost-savingstrategy. Catheter Cardiovasc Interv 2013; 81: 15-23.

19. Ruzsa Z, Ungi I, Horváth T, et al. Five-year experience with transradial coronary angioplasty in ST-segment-elevation myocardial infarction. Cardiovasc Revasc Med 2009; 10: 73-9.

20. Maciejewski D, Tekieli $九$, Kabłak-Ziembicka A, et al. Transradial approach for vertebral artery stenting. Adv Interv Cardiol 2015; 11: 32-6.

21. Folmar J, Sachar R, Mann T. Transradial approach for carotid artery stenting: a feasibility study. Catheter Cardiovasc Interv 2007; 69: 355-61.

22. Madden NJ, Calligaro KD, Zheng H, et al. Outcomes of brachial artery access for endovascular interventions Ann Vasc Surg 2019; 56: 81-6.

23. van Mook WN, Rennenberg RJ, Schurink GW, et al. Cerebral hyperperfusion syndrome. Lancet Neurol 2005; 4: 877-88.

24. Abou-Chebl A, Yadav JS, Reginelli JP, et al. Intracranial hemorrhage and hyperperfusion syndrome following carotid artery stenting: risk factors, prevention, and treatment. J Am Coll Cardiol 2004; 43: 1596-601.

25. Lieb M, Shah U, Hines GL. Cerebral hyperperfusion syndrome after carotid intervention: a review. Cardiol Rev 2012; 20: 84-9.

26. Moulakakis KG, Mylonas SN, Sfyroeras GS, Andrikopoulos V. Hyperperfusion syndrome after carotid revascularization. J Vasc Surg 2009; 49: 1060-8.

27. Huibers AE, Westerink J, de Vries EE, et al. Cerebral hyperperfusion syndrome after carotid artery stenting: a systematic review and meta-analysis. Eur J Vasc Endovasc Surg 2018; 56: 322-33.

28. Khan M, Qureshi Al. Factors associated with increased rates of post-procedural stroke or death following carotid artery stent placement: a systematic review. J Vasc Interv Neurol 2014; 7: 11-20.

29. Fairman R, Gray WA, Scicli AP, et al. The CAPTURE registry: analysis of strokes resulting from carotid artery stenting in the post approval setting: timing, location, severity, and type. Ann Surg 2007; 246: 551-6.

30. AbuRahma AF, DerDerian T, Hariri N, et al. Anatomical and technical predictors of perioperative clinical outcomes after carotid artery stenting. J Vasc Surg 2017; 66: 423-32.

31. Schneider PA, Levy E, Bacharach JM, et al. A first-in-human evaluation of a novel mesh-covered stent for treatment of carotid stenosis inpatients at high risk for endarterectomy: 30-day results of the SCAFFOLD trial. J Am Coll Cardiol Intv 2018; 11: 2396-404.

32. Machnik R, Paluszek P, Tekieli Ł, et al. Mesh-covered (Roadsaver) stent as a new treatment modality for symptomatic or high-risk carotid stenosis. Adv Interv Cardiol 2017; 13: 130-4. 